\title{
Management of a complicated triathlete's bilateral iliac artery endofibrosis
}

\begin{abstract}
Introduction: Iliac artery endofibrosis (IAE) is an uncommon disease, poorly studied pathology with devastating effects and different therapeutic approaches affecting young people who practise intensive sports, especially cyclists. The evolution of the process not only depends on the diagnosis and therapeutic action, but also on the acceptance and attitude of the patient and subsequent professional guidance.

Case description: This is the case description of a professional triathlon athlete that had one previous iliac surgical revascularization for an IAE Iliac and was admitted in our department five times with subacute lower limb ischemia affecting both legs between 2013 and 2016. Clinical findings and image tests are reported, as well as medical procedures performed. Indications based on clinical, functional and imaging ratings were clear, but his professional activity was not completely abandoned. Finally, after four endovascular procedures with good immediate results, he was warned of the seriousness of the process since the etiopathogenic reason. At the present moment patient is asymptomatic, under routine controls, working as successful triathlon coach.

Discussion and conclusion: The fact that an external mechanical stress is the reason of repeated iliac artery injury suggests that an open surgical approach correcting the external muscular compression or arterial deformation should be a definitive but also aggressive solution according to literature. However, endovascular procedures and new endovascular devices are an increasingly promising option with a very low surgical risk. No matter the revascularization performed, the persistence of sports intensive practice carries a high risk of recurrence. Sport practise cessation is mandatory in some cases in order to assure revascularization long-term patency, but also a well conducted professional orientation is needed to complete the therapeutic action.
\end{abstract}

Keywords: lower limb ischaemia, triathlon, pain
Volume 5 Issue 3 - 2018

\author{
Joan Fité,' Jaime Félix Dilmé,' Jordi Villalba,' \\ Olga Peypoch,' Luis Til, ' José Román \\ Escudero,' Franchek Drobnic ${ }^{2}$ \\ 'Department of Vascular and Endovascular Surgery, Hospital de \\ la Santa Creu i Sant Pau, UAB, CIBER CV, Spain \\ ${ }^{2}$ Department of Sports Medicine, Girsane, Medical Services FC \\ Barcelona
}

Correspondence: Franchek Drobnic, Department of Sport Research, CAR, Avda. Alcalde Barnils 3-5, 08174 Sant Cugat de Vallés, Spain, Medical Services FC Barcelona, Tel +3493589 15 72,Email drobnic@car.edu

Received: April 25, 2018 | Published: May 17, 2018

\section{Introduction}

Muscular injury is the most frequent reason for effort-induced leg pain in athletes and in young active population in general, but the persistence of effort-induced pain and effort claudication in time despite correct treatment should make clinicians consider other less common diagnoses. ${ }^{1}$ In the early nineties a form of iliac artery disease affecting young and apparently healthy athletes was first described, and many years later it was defined as iliac artery endofibrosis (IAE). The low prevalence of the disease and the variety of clinical presentations make this disease very unknown and poorly studied, despite the improvement of image tests, and there are no standard treatment protocols. Literature revision shows very few publications on iliac artery endofibrosis, being the majority of them short case series with different therapeutic options without enough evidence to establish the best choice. Furthermore, publications regarding aetiology and diagnosis of the disease are based on very few patients, thus raising theories about the origin of the IAE that should be verified by clinical studies including a higher amount of patients., ${ }^{2,3}$ Most are men, cyclists, but also described in many other sports, probably due to a combination of genetic factors and the greater number of men practising high-intensity sports. ${ }^{1,3,4}$ From an anatomical point of view, it is caused by a thickening of the iliac artery intimae that causes hemodynamic stenosis. The reason of this local fibrosis is a repeated mechanical trauma on the artery and its secondary inflammatory response. It usually affects the external iliac artery, but it has also been described in common iliac artery or even common femoral arteries. Clinical signs vary according to the stenosis level, and this stenosis can also be complicated with adjacent thrombosis and arterial acute or subacute occlusion, focal artery wall dissection or even local atherosclerotic changes in chronic cases. Both unilateral and bilateral cases have been reported, probably in relation with the type activity., Histological studies of IAE show subintimal dense loose connective tissue deposits rich in elastin and actin-positive smooth muscle cells, making a clearly different disease when compared to atherosclerosis or other vascular diseases histology. Instead, atherosclerotic disease shows more densely packed collagen deposits; extensive calcification is present in almost all cases, while in endofibrotic disease this is uncommon. Inflammatory cells are rarely present in iliac endofibrosis, but it is a common feature in atherosclerotic lesions, specially involving macrophages. Both endofibrotic and atherosclerotic areas seem to have a prothrombotic effect on blood flow. There are few studied involving immunohistochemical staining of iliac artery samples and results can be biased by important median age difference between endofibrosis patients and atherosclerotic patients. ${ }^{6}$

Aetiology remains unclear. It has been stated that intimal fibrosis and hyperplasia is caused by an external repeated traumatism produced during the exercise activity, or by hypertrophic psoas muscle contraction and artery external compression. It has also been reported 
that iliac artery collateral vessels to the psoas muscle may affix the central portion of the external iliac artery and ankle forced flexion in some sports may stretch the distal part of the artery, thus causing gradual artery elongation and intimal endofibrosis. It is empirically assumed that some patients may have a basally elongated iliac artery, being predisposed to early iliac endofibrosis when practising sports, as well as there could be patients with some uncommon metabolic abnormalities. ${ }^{1,4,6,7}$

Iliac artery endofibrosis appears with clinical signs and symptoms of various characteristics and intensities, and this is why IAE is usually misdiagnosed. Symptoms can range from muscle claudication signs in the affected limb to subacute or acute limb ischemia due to complete obliteration of the iliac artery. When there is only endofibrotic iliac stenosis, there is calf and proximal muscle pain in moderate-intense efforts appearing gradually, but there are no rest clinical signs and even peripheral pulses are present at rest. ${ }^{1,3,8}$ Decrease of femoral systolic flow speed in duplex ultrasound evaluation is usually present, but when acute thrombosis or dissection of the iliac artery lead to complete arterial occlusion, ischemic signs are more clear, with absence of all pulses in the affected limb and short distance claudication or even paresthesia, pallor and rest pain., ${ }^{1,4}$

Properly trained clinicians and vascular surgeons can easily perform a correct diagnosis with an appropriate anamnesis and physical examination. Ankle-brachial index (ABI) after moderate intensity effort and femoral doppler flow evaluation have an elevate sensitivity and specificity to identify iliac obstruction. To assess anatomic details and the exact position of the stenosis, literature suggests magnetic resonance angiography and digital subtraction angiography as the gold standard image tests. Both tests can identify stenosis, arterial kinking and elongation, and collateral arteries that may tether the iliac artery to the psoas muscle. Angiography with limb flexion compared to rest position may clarify functional reasons of iliac endofibrosis. Some studies reveal that duplex ultrasound can detect both external iliac artery stenosis and excessive artery length with similar sensitivity than digital subtraction angiography, but angiography is more reliable to evaluate small muscular arteries tethering the external iliac artery. ${ }^{9}$

Treatment should be multidisciplinary and there is not a clearly defined therapeutic protocol. ${ }^{10}$ Medical and conservative treatment should be the first option, especially in non-critical iliac stenosis with mild symptoms. Atherosclerotic factors should be treated according to clinical guidelines if present. Intensive sport activity should be immediately stopped or at least changed into a different sport practice. Only if symptoms persist, surgical options should be taken into account. The role of simple angioplasty or stent angioplasty is not clear. Short term results are promising but the anatomical and mechanical ethiopathogenesis causing the IAE are not solved, and mid-term recurrence due to local intimal hyperplasia occurs. The gold standard technique is open surgical repair, consisting of a complete iliac artery dissection in order to separate any constrictive surrounding tissue and to disconnect $\mathrm{m}$. psoas major arterial branches. In cases of important artery elongation or very severe focal obstruction, endarterectomy and arterial patch with surgical artery shortening have been reported, as well as a prosthetic bypass surgery. These techniques seem to be more definitive but surgical risks are significantly higher and postoperative recovery is more complicated than in endovascular procedures. The low prevalence of IAE, absence of clinical trials comparing tests or treatment options and lack of long-term follow-up results make it impossible to establish protocols. Longer case series and clinical trials with long-term results should be performed. ${ }^{1,3,11,12}$

\section{Case description}

This is the case of a 28 years old professional triathlon athlete visited in our vascular surgery department in February 2013 due to the progressive appearance of ischemic left lower limb symptoms since two months before. Left limb claudication appeared after daily training, with transient foot paresthesia and pallor. These symptoms had gradually increased disabling him for competition. His medical history revealed a left iliofemoral prosthetic bypass performed in 2007 , for similar symptoms. He had no other prior surgeries and no chronic or significant diseases were reported. At the moment he was under no medical treatments. Physical exploration showed pulselessness in the left lower limb with discrete foot pallor, and distal pulses were present in the right lower limb. Rest pain was absent, sensitivity and motility were preserved, but he did not tolerate any intensive physical effort in left lower limb with significant calf pain and ankle claudication. Ankle brachial index (ABI) was performed with a normal result in the right lower limb and 0.61 in the left pedial artery. Standardized treadmill test revealed pain and claudication with decrease of left leg blood pressures after $470 \mathrm{~m}$ (walking at $3 \mathrm{~km} / \mathrm{h}$ with a regular $10 \%$ uphill). An angio CT scan was performed, revealing complete occlusion of both the left external iliac artery (EIA) and the iliofemoral bypass graft, with patency of the common femoral artery in the left lower limb. In the right lower limb there were no pathological findings. Sport cessation was recommended and $100 \mathrm{mg}$ salicylic acid was prescribed as antiaggregant. External iliac artery endofibrosis was suspected, and he was planned for surgery in April 2013. An endovascular procedure was performed under local anaesthesia. Native EIA was revascularized from a retrograde femoral approach, and a pharmacologic balloon angioplasty was performed with optimal angiographic results. He was discharged 24 hours later with both $100 \mathrm{mg}$ aspirin and $75 \mathrm{mg}$ clopidogrel antiaggregation and the strong recommendation to stop high-intensity training that could have caused the external iliac endofibrosis and subsequent thrombosis. One month after the revascularization a control angio CT scan was performed with normal results, and two months later a duplex ultrasound routine evaluation was done. Six months after the angioplasty, clopidogrel was stopped, keeping $100 \mathrm{mg}$ aspirin daily as a chronical treatment, but, in spite of the recommendations, the trainings did not stop.

One year later he was visited for the appearance of ischemic symptoms in the right lower limb, calf claudication, pulselessness and subtle pallor, with no critical ischemia signs. An emergency duplex ultrasound evaluation showed a pathological Doppler curve and lowered systolic blood flow speed in the right femoral artery thus suggesting iliac stenosis. With the diagnostic orientation of a complicated right EIA endofibrosis, low molecular weight heparin was prescribed to avoid progressive thrombosis and a new angio CT scan was performed. The CT scan demonstrated an acute thrombosis of the right EIA, with patency of the previously revascularized left EIA, and an endovascular revascularization was programmed (Figure 1) (Figure 2). 
Through a retrograde right femoral access a pharmacologic balloon angioplasty was performed in both right common and external iliac arteries, with excellent angiographic results. All right lower limb pulses were present after the procedure, and he was discharged under double antiaggregant therapy and the medical recommendation to limit sport practice, cycling and running absolutely. Posterior clinical controls were normal, with a normal postoperative ABI, normal angio $\mathrm{CT}$ scan one month after the revascularization and successive normal duplex ultrasound evaluations.

In April 2015, the patient was admitted again in our department due to the appearance in the last $24 \mathrm{~h}$ of a short distance right lower limb claudication. In this case, the right limb presented a positive femoral pulse, but right popliteal and distal pulses were absent. Left lower limb evaluation was completely normal. The patient confirmed he kept on training and participating in elite triathlon competitions despite our recommendations. Angio CT scan confirmed a new thrombosis, now at the right EIA. Thrombus images and clinical acute presentation indicated there was a fresh, non-fibrotic thrombus in the occluded artery. A hybrid approach was selected. Through a surgical open femoral access, distal femoral control to prevent distal embolism and proximal thrombectomy with Fogarty catheters were performed. In control angiography a short stenosis was observed in the right EIA, requiring a pharmacologic balloon angioplasty with angiographic optimal final result (Figure 3A) (Figure 3B). He was discharged again $24 \mathrm{~h}$ after the procedure with a normal physical evaluation and double antiaggregant were prescribed. In order to optimize medical treatment, hematologic evaluation was performed in order to evaluate any possible thrombophilic disease. All results were negative. Even though the patient had been informed that intensive sports were the aetiology of the iliac endofibrosis and the ischemic events, he did not stop training and competing. All clinical controls were normal until May 2016, when a new middle distance claudication appeared affecting the right lower limb. Image tests confirmed acute thrombosis of the right EIA, and an endoluminal thrombus aspiration and pharmacologic angioplasty of the right EIA were performed. Functional test and images confirm the resolution of the process (Figure 4). Since this procedure, patient seemed to understand and stopped intensive sport practise and remained asymptomatic in ambulatory controls. Chronic double antiaggregation was recommended. But, the athlete, searching for a diagnosis or therapeutic guidelines that better fit his sporting desires, requested an assessment from the Medical Services at the Olympic Training Center (OTC). In June 2016, in the OTC, during a cycle ergometer exercise test simulating a training session, the ischemic symptoms, right thigh pain, lack of pulse and pale foot, reappeared at the 15 minute at steady state power of 225 watts and 165 beats $/ \mathrm{min}$. The circulation recovered very slowly and the pulse did not appear until the 5 minutes of rest. That exercise response demonstrated to the athlete, finally, that an acute ischemic process in response to exercise exists, even while subject maintained drug therapy, previous good vascular diameter and by peripheral doppler scan ultrasound, without changes after short and maximal isometric contraction.

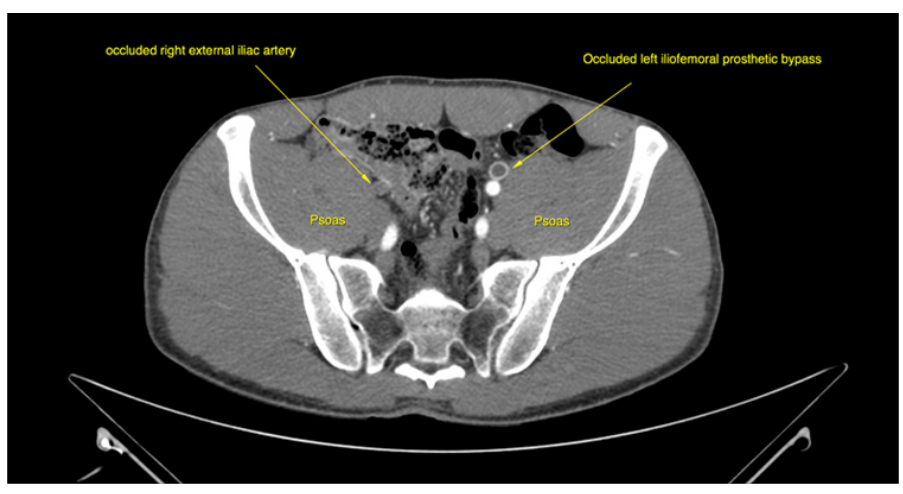

Figure I Transversal CT scan diagnostic figure showing right EIA occlusion.
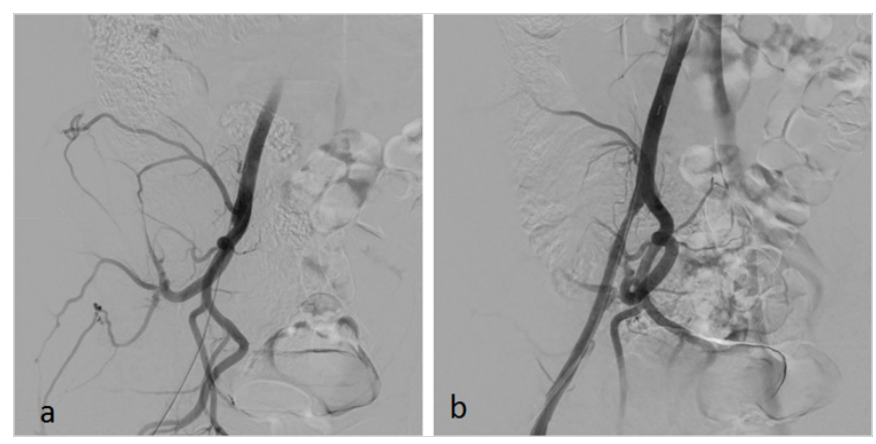

Figure 3 Guide wire over passing the right EIA occlusion (a) with the angiographic final revascularization result (b).

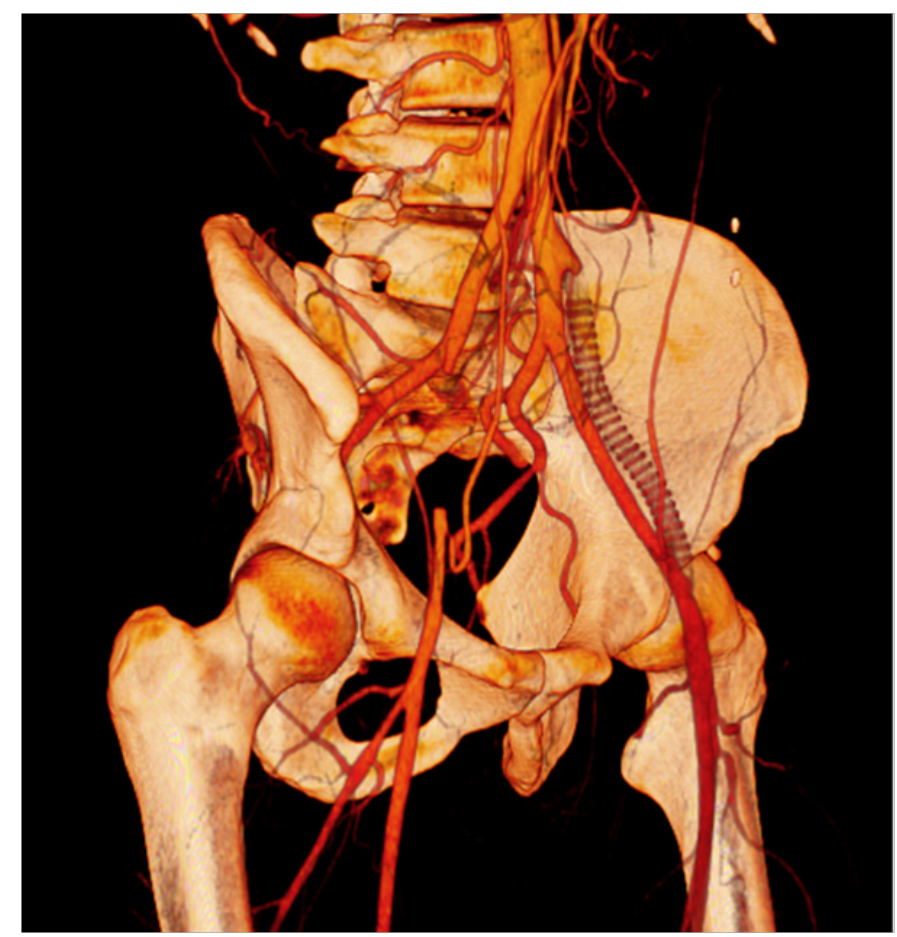

Figure $2 \mathrm{CT}$ scan oblique reconstruction showing occlusion of the right EIA, the occluded iliac prosthetic graft and patency of the native left iliac arteries after revascularization. 


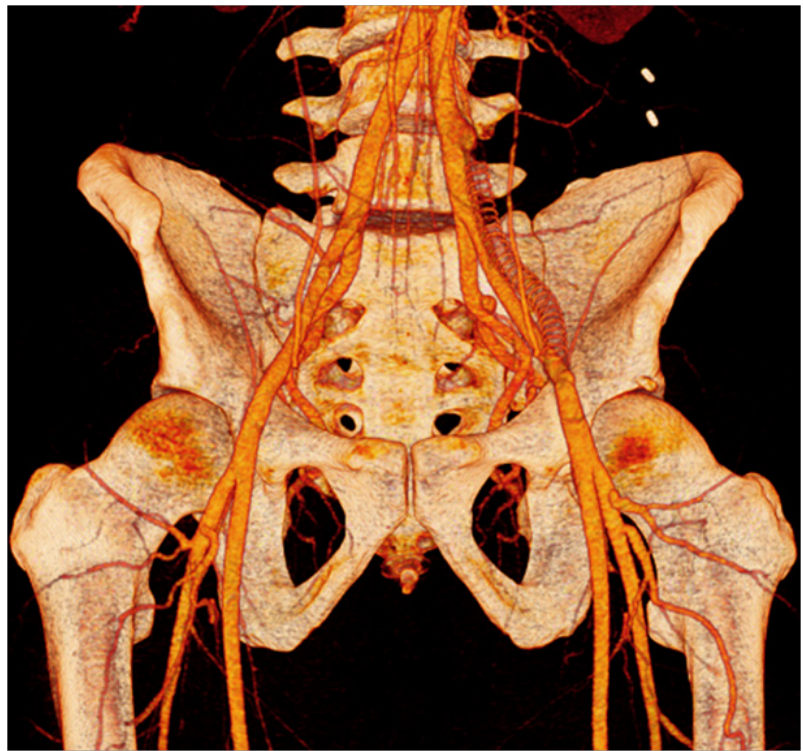

Figure 4 Coronal reconstruction of the last control CT scan.

\section{Discussion}

Iliac artery endofibrosis is a challenging pathology because it affects very young people with possible devastating effects but aetiology and therapeutic options remain uncertain. Even though literature raises the idea that open surgical repair is the most definitive option, we consider treatment indication should be individualised in all patients. In this case there are three factors that made our department decide to apply endovascular procedures. The first reason was the existence of a failed prior open surgery. When revising the angioCT scan and angiography images, we identified an important psoas and lumbar muscular hypertrophy that seemed to be the external mechanical reason of the bypass graft obliteration due to an external compression. Furthermore, in angiographic images no significant psoas artery collaterals were observed, thus suggesting that there was no EIA tethering to be solved, and iliac artery elongation was not notorious. It therefore seemed that there were no significant anatomical factors that would benefit from open surgical correction. Instead, in such a young and active patient open surgery carried higher complication rates and longer recovery periods after surgery. Endovascular options are technically feasible, with low and minor complication rates when compared to open iliac revascularizations, and even though these seem to have a limited patency in time, this case shows that endovascular iliac revascularizations are easily reproducible with optimal immediate results and minimal hospital admittance. Nevertheless, either endovascular or surgical procedures fail if the mechanic origin of the iliac artery endofibrosis persists. Our patient underwent intensive clinical controls and a correct medical treatment to enhance endovascular revascularization patency and to prevent thrombotic events, but there was no sport cessation. According to clinical history, acute symptoms usually appeared after competitions of intensive trainings. It seems that this patient had bilateral EIA endofibrosis due to triathlon competitions and training (including intensive cycling sessions), probably due to the external continuous mechanic trauma of hypertrophic lumbar muscles adjacent to the IAE (Figure 5). In this context, some especially intensive efforts involving important dehydration may have led to complication of the endofibrotic area causing the EIA spasm and thrombosis.

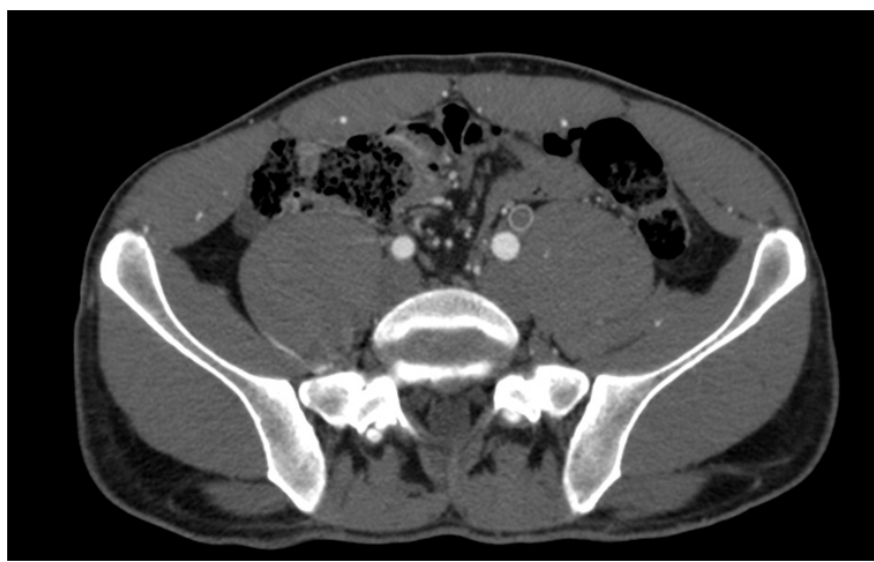

Figure 5 CT scan transverse image showing $\mathrm{m}$. psoas massive hypertrophy and anatomical relation to iliac arteries.

For a successful and easy endovascular repair, treatment should be applied short after clinical signs appear. This way we avoid local thrombosis to become fibrotic and harder to surpass with endovascular materials. Both femoral and brachial accesses are suitable and minimally invasive for iliac revascularizations. Being the IAE the only artery affected, according to experience on atherosclerotic peripheral arteriopathy we would recommend pharmacologic ballon angioplasty, reserving stent implantation only in case of persistent stenosis or flowlimiting focal dissection. In all cases, double antiaggregation seems recommendable.

In cases in which no artery elongation or significant tethering is detected, we suggest endovascular procedures as a first and low-risk choice for IAE patients. Instead, if there are correctible anatomical factors explaining the IAE, or endovascular procedures have already been performed and failed despite sport cessation, open surgical revascularization should be taken into account. Despite our efforts, it was impossible to convince the patient to stop sport competition after the first revascularizations we performed. The reproducibility and short recovery period of endovascular procedures probably made this disease appear more harmless to the eyes of the patient. Iliac endofibrosis patients are usually really young patients with high life expectancy. Endovascular techniques have very good results in iliac arteries and new devices would even probably improve patency and possibilities, but all clinician efforts should be directed to understand the patient and look the best way to convince him to stop practising intensive sports. Otherwise, both endovascular and surgical repair are committed to fail. Otherwise, the persistence of sports intensive practise carries long probabilities of reintervention, major comorbilities and long-term invalidation.

\section{Acknowledgements}

None.

\section{Conflict of interest}

We declare that the results of the case presentation are presented clearly, honestly, and without fabrication, falsification, or inappropriate data manipulation, and that the results of the present study do not 
constitute endorsement by ACSM. The case it is presented with the informed consent by the patient, in order to help other athletes in reaching the best medical decision and personal commitment that guarantees their health.

\section{References}

1. Peach G, Schep G, Palfreeman R, et al. Endofibrosis and kinking of iliac arteries in athletes: a systematic review. Eur J Vasc Endovasc Surg. 2012;43(2):208-217.

2. Chevalier JM, Enon B, Walder J, et al. Endofibrosis of the external iliac artery in bicycle racers, an unrecognised pathological state. Ann Vasc Surg. 1986;1(3):297-303.

3. Lim CS, Gohel MS, Shepherd AC, et al. Iliac artery compression in cyclists: mechanisms, diagnosis and treatment. Eur J Vasc Endovasc Surg. 2009;38(2):180-186.

4. Ford SJ, Rehman A, Bradbury AW. External iliac endofibrosis in endurance athletes: a novel case in an endurance runner and a review of the literature. Eur J Vasc Endovasc Surg. 2003;26(6):629-634.

5. Franco A, Rigberg DA, Ruehm SG. Bilateral common iliac artery endofibrosis in a recreational cyclist: case report and review of the literature. Ann Vasc Surg. 2016;35:203.e11-5.

6. Vink A, Bender MH, Schep G, et al. Histopathological comparison between endofibrosis of the high-performance cyclist and atherosclerosis in the external iliac artery. J Vasc Surg. 2008;48(6):1458-1463.
7. Kral CA, Han DC, Edwards WD, et al. Obstructive external iliac arteriopathy in avid bicyclists: new and variable histopathologic features in four women. J Vasc Surg. 2002;36(3):565-570.

8. Schep G, Bender MH, Schmikli SL, et al. Recognising vascular causes of leg complaints in endurance athletes. Part 2. Int J Sports Med. 2002;23(5):322-328.

9. Alimi YS, Accrocca F, Barthèlemy P, et al. Comparison between duplex scanning and angiographic findings in the evaluation of functional iliac obstruction in top endurance athletes. Eur J Vasc Endovasc Surg. 2004;28(5):513-519.

10. INSITE Collaborators (INternational Study group for Identification and Treatment of Endofibrosis). Diagnosis and management of iliac artery endofibrosis: results of a delphi consensus study. Eur J Vasc Endovasc Surg. 2016;52(1):90-98.

11. Giannoukas A, Berczi V, Anoop U, et al. Endofibrosis of iliac arteries in high performance athletes: diagnostic approach and minimally invasive endovascular treatment. Cardiovasc Interv Rad. 2006;29(5):866-869.

12. Bender MHM, Schep G, Bouts SW, et al. Endurance athletes with intermittent claudication caused by iliac artery stenosis treated by endarterectomy with vein patch-short-and mid-term results. Eur J Vasc Endovasc Surg. 2012;43(4):472-477. 